\title{
Antineutrino Physics at MINOS
}

\author{
A. I. Himmel for the MINOS Collaboration \\ Lauritsen Laboratory, California Institute of Technology, 1200 E. California Blvd., Pasadena, CA
} 91125, USA

\begin{abstract}
We present two new measurements of antineutrino properties based on a data sample corresponding to $3.2 \times 10^{20}$ protons-on-target, exploiting MINOS' unique ability to distinguish positive and negative muons and thus se parate charged current neutrino and antineutrino interactions event-by-event. The first measurement takes advantage of the $6 \%$ antineutrino component of the NuMI neutrino beam to measure antineutrino oscillations between the near and far detectors. We observe 42 events at the far detector with an expectation of $58.3 \pm 7.6$ (stat.) \pm 3.6 (syst.) assuming CPT-conserving oscillations, excluding $\left(5.0<\Delta \bar{m}^{2}<81\right) \times 10^{-3} \mathrm{eV}^{2}$ at $90 \%$ confidence at maximal mixing. We also present a search for neutrino-antineutrino transitions $v_{\mu} \rightarrow \bar{v}_{\mu}$, which would result in an excess of antineutrino events in the Far Detector relative to the rate expected from the intrinsic antineutrino component in the neutrino beam. We observe no excess and set a limit of 0.026 on the transition probability at $90 \%$ confidence.
\end{abstract}

Keywords: Antineutrinos, CPT, Oscillations, Transitions, Long Baseline, MINOS

PACS: $14.60 . \mathrm{Pq}$

MINOS is a long baseline neutrino oscillation experiment designed to precisely measure the atmospheric mixing parameters, $\Delta m^{2}$ and $\sin ^{2}(2 \theta)$. It consists of the NuMI neutrino beam at Fermilab and two detectors: a near detector located $1 \mathrm{~km}$ from the target and a far detector in Soudan, MN located $735 \mathrm{~km}$ from the target. The detectors are functionally identical tracking, sampling calorimeters made up of alternating layers of $2.54 \mathrm{~cm}$ thick iron and $1 \mathrm{~cm}$ thick plastic scintillator and they both have an approximately $1.3 \mathrm{~T}$ toroidal magnetic field. The far detector is $5.4 \mathrm{ktons}$ in mass and is located $700 \mathrm{~m}$ below ground while the near detector is $1.0 \mathrm{kton}$ in mass and is located $100 \mathrm{~m}$ underground [1].

$\mathrm{NuMI}$ is an intense beam made up primarily of muon neutrinos $(91.7 \%)$ with small additional components of muon antineutrinos $(7.0 \%)$ and electron neutrinos and antineutrinos $(1.3 \%)$. It is produced by colliding $120 \mathrm{GeV}$ protons from Fermilab's Main Injector onto a thick, segmented graphite target creating mesons that are then focused by two magnetized horns and allowed to decay in a $675 \mathrm{~m}$ decay pipe. By focusing the positive mesons, the neutrino flux is enhanced at the expense of the antineutrino flux. The majority of the antineutrinos in MINOS come from pions and kaons that travel "neck-to-neck" down the center of the two horns and thus avoid being defocused with the remaining significant fraction coming from interactions with the other material in the beamline. ${ }^{1}$

We use the MINOS antineutrino sample to make two measurements. The first uses

${ }^{1}$ This downstream production occurs for both neutrinos and antineutrinos; however, it is only a significant component for antineutrinos since the otherwise much larger target flux is defocused. 


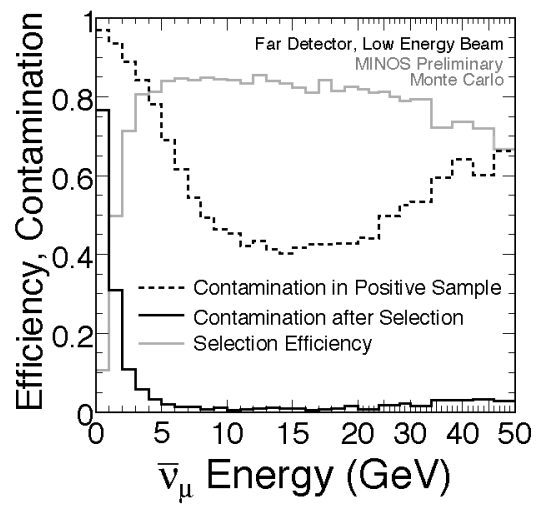

(a)

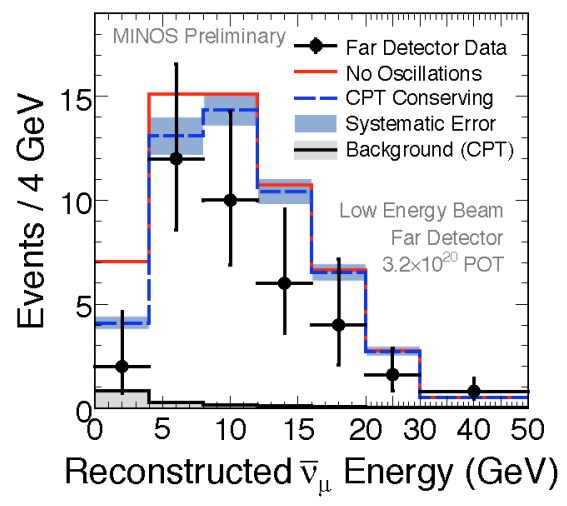

(b)

FIGURE 1. (a) The efficiency and contamination of the NuMuBar sample at far detector. The dashed line shows the contamination in the sample of all events with positive reconstructed tracks. The solid lines show the efficiency and contamination after applying all selection cuts. (b) The far detector data spectrum (black markers) plotted with predicted spectrum assuming CPT-conserving oscillations (dashed) and assuming no oscillations (solid). The error bars on the data are statistical and the error bars on the prediction are systematic.

the intrinsic beam flux to search for antineutrino disappearance at the far detector and thus measure the antineutrino atmospheric mixing parameters, $\Delta \bar{m}^{2}$ and $\sin ^{2}(2 \bar{\theta})$. The second measurement looks for antineutrino appearance at the far detector above the intrinsic beam flux, indicating transitions[2] from the neutrinos which are known to disappear[3]. We parameterize this appearance by supposing that there is some probability, $\alpha$, that a disappearing neutrino will transition to an antineutrino: $P\left(v_{\mu} \rightarrow \bar{v}_{\mu}\right)=$ $\alpha \sin ^{2}(2 \theta) \sin ^{2}\left(1.27 \Delta m^{2} L / E\right)$. This appearance has a characteristic signature in the energy spectrum because the neutrino spectrum is sharply peaked below the average antineutrino energy.

Antineutrino events in the MINOS detectors are characterized by long $\mu^{+}$tracks that curve towards the outside of the detectors with small hadronic showers at the vertex. However, just selecting events that are reconstructed as positive gives a sample that is dominated by background because the antineutrinos make up so few of the detector events $(7.0 \%$ of the near and $6.4 \%$ of the far) as can be seen in the dashed line in Figure 1a. There are two classes of background: neutral current events with a fake muon track and charged current muon neutrino events where the sign of the muon is misidentified. Two types of selectors are used to eliminate this background: a likelihoodbased charged current/neutral current discriminator and two charge sign selectors. The $\mathrm{CC} / \mathrm{NC}$ discriminator uses track $d E / d x$, event length, and the relative amount of energy in the track and shower while the charge sign selectors use the relative error in the measured curvature and the relative angle between the beginning and end of the track. After applying these selections, we have an overall relative efficiency of $82 \%$ and a purity of $97 \%$ at the Far Detector. 


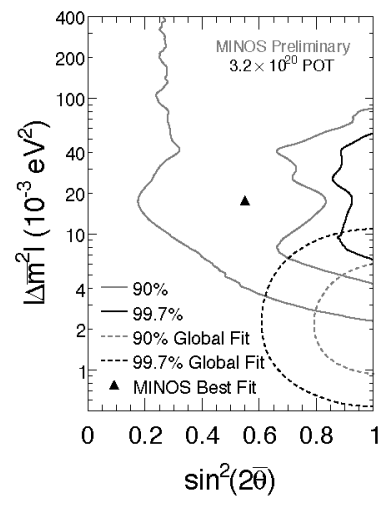

(a)

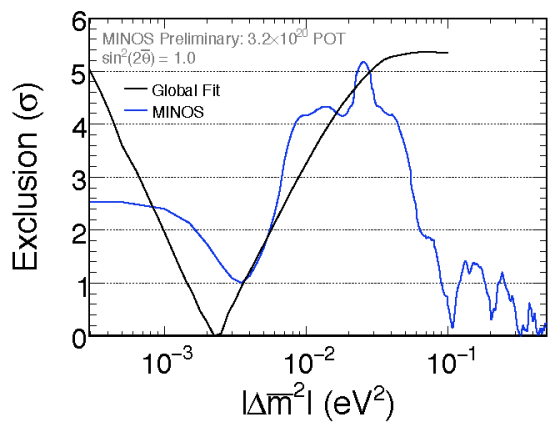

(b)

FIGURE 2. (a) The two-dimensional best fit contours for antineutrino oscillations. The solid lines are based on the MINOS data and the dashed lines are based on a fit to all other global data[4]. (b) The one-dimensional exclusion contour for $\left|\Delta \bar{m}^{2}\right|$ at maximal mixing.

While the near and far detectors are functionally identical to reduce systematics, they do not have identical spectra: the near detector is so close to the decay pipe, it covers a much wider solid angle and sees an extended source of neutrinos while the far detector sees a point source with only very forward-going decays. Using knowledge of the beamline geometry and the kinematics of pion and kaon decay, a 'beam matrix' is created that relates a particular near detector energy to a distribution of far detector energies. Using this technique we can 'extrapolate' the near detector spectrum and obtain a far detector prediction.

We performed a blind analysis on this data set: all selections and systematics were determined before looking at the far detector data. The measured far detector spectrum can be seen in Figure $1 \mathrm{~b}$ along with two predictions: CPT-conserving (dashed) and no oscillations (solid). 42 events were observed with an expectation of $58.3 \pm 7.6$ (stat.) \pm 3.6 (syst.) with CPT-conserving oscillations and $64.6 \pm 8.0$ (stat.) \pm 3.9 (syst.) with no oscillations. The probability of getting 42 events or fewer assuming the true underlying spectrum is CPT-conserving is $5 \%$.

Figure 2a shows the contours obtained by fitting the far detector data to the oscillations hypothesis; the MINOS results (solid) are shown along with the fit to all other global data (dashed) [4]. These contours are consistent at $90 \%$ confidence with the neutrinos and antineutrinos having the same oscillation parameters. The best fit point is at high $\Delta \bar{m}^{2}$ because the deficit in the data is at higher energies (the oscillation minimum for the neutrino mixing parameters is at $1.4 \mathrm{GeV}$ ). Since the limited statistics of our data do not allow us to constrain the mixing angle well, we also performed a one-dimensional fit for $\Delta \bar{m}^{2}$ at maximal mixing (the global fits constrain $\sin ^{2}(2 \bar{\theta})>0.92$ at $68 \%$ ). This is shown in Figure 2b. Doing so, we can exclude oscillations with $\left(5.0<\Delta \bar{m}^{2}<81\right) \times 10^{-3} \mathrm{eV}^{2}$ at $90 \%$ confidence at maximal mixing.

The far detector data was also fit to the transitions hypothesis. There was no observed 
antineutrino appearance placing an upper limit of $\alpha<0.026$ on the neutrino to antineutrino transition probability at $90 \%$ confidence.

After the summer shutdown at Fermilab NuMI will be running in dedicated antineutrino mode with the polarity of the magnetic focusing horns reversed. Running in this mode overcomes the two main difficulties of studying antineutrino oscillations: the low statistics and higher average energy which conspire to give very few events at low energies where oscillations are expected to be observed.

By reversing the current in the two focusing horns the negative pions and kaons are focused, producing an intense muon antineutrino beam. In this running mode we expect only about one third of the events in the focusing peak per a given number of protons on target due to a combination of lower negative meson production cross-sections and lower antineutrino interaction cross-sections. Even so, with one fiscal year of running $\left(2 \times 10^{20}\right.$ protons-on-target) a five sigma observation of antineutrino disappearance can be made.

We present two new measurements using the MINOS antineutrino sample. The magnetized detectors allow antineutrinos to be separated from neutrinos on an event-byevent basis. At the far detector we observe 42 events with an expectation of $58.3 \pm$ 7.6 (stat.) \pm 3.6 (syst.) assuming CPT-conserving oscillations. Using the intrinsic antineutrinos in the NuMI beam we exclude antineutrino oscillations with $\left(5.0<\Delta \bar{m}^{2}<\right.$ $81) \times 10^{-3} \mathrm{eV}^{2}$ at $90 \%$ confidence at maximal mixing. We also observe no evidence for antineutrino appearance and limit the probability of neutrino-to-antineutrino transitions to $\alpha<0.026$ at $90 \%$ confidence.

\section{ACKNOWLEDGMENTS}

We would like to express our gratitude to the many Fermilab groups who provided technical expertise. We also gratefully acknowledge financial support from Department of Energy, Science and Technology Facilities Council (UK), National Science Foundation and thank the University of Minnesota and the Minnesota Department of Natural Resources for hosting us.

\section{REFERENCES}

1. P. Adamson, et al., Phys. Rev. D 77, 072002 (2008), 0711.0769.

2. P. Langacker, and J. Wang, Phys. Rev. D 58, 093004 (1998).

3. P. Adamson, et al., Phys. Rev. Lett. 101, 131802 (2008), 0806.2237.

4. M. Gonzalez-Garcia, and M. Maltoni, Phys. Rept. 460, 1-129 (2008). 